\title{
22. METEORS AND INTERPLANETARY DUST (MÉTÉORES ET LA POUSSIÈRE INTERPLANÉTAIRE)
}

PRESIDENT: B. A. Lindblad.

VICE-PRESIDENT: I. Halliday.

ORGANIZING COMMITTEE: A. F. Cook, W. G. Elford, T. R. Kaiser, L. Kresák, B. J. Levin, R. E. McCrosky, F. F. Verniani.

\section{INTRODUCTION}

The content and format of this report differ somewhat from the previous reports. The changed mandate of our commission implies that the physical study of meteorites is covered in the report of Commission 15. The emphasis in the present report has been more on a critical review than on a complete bibliographic survey. The subject matter has been divided into nine topics, each assigned to a particular author. The President has acted as editor and assumes full responsibility for any errors or omissions. The various authors thank those colleagues who responded to the request for reprints and information on current activities. We are in particular indebted to Dr P. B. Babadzhanov for a report on activities in the field of meteors and interplanetary dust in the U.S.S.R., from which we have inserted references in the appropriate sections below. Whenever possible the reference system of Astronomy and Astrophysics Abstracts has been used. Other references are given at the end of each section, except for articles appearing in certain IAU Colloquia:

Colloq. 13, 'The Evolutionary and Physical Problems of Meteoroids', C. L. Hemenway,

A. F. Cook, and P. M. Millman (eds.), NASA SP-319, Washington, 1973.

Colloq. 22, 'Asteroids, Comets, Meteoric Matter', C. Cristescu, W. J. Klepezynski and B. Millet (eds.), Edit. Acad. Rep. Soc. Rumania, Bucarest, 1974.

Colloq. 31, 'Interplanetary Dust and Zodiacal Light', (Lecture Notes in Physics, No. 48), Springer-Verlag, Heidelberg, 1976 (in press).

Before beginning the detailed report it seems appropriate to express our feelings of deep regret at the loss of the distinguished meteor astronomer and organizer of amateur meteor observations, C. P. Olivier (1884-1975). Another great loss for meteor research was the death of I. S. Astapovich.

\section{GENERAL}

During the last three years there has been considerable activity in the field of meteor physics and astronomy. An important bibliographic work is the publishing of The Collected Papers of Fred L. Whipple (13.003.153). A book on Radio Meteor Investigations of the Upper Atmosphere has been published by Babadzhanov and co-workers (13.003.028). Review papers on interplanetary material have been published by Dohnanyi (08.106.009) and Millman $(10.105 .019,11.106 .008)$. Other important review papers on interplanetary dust were presented at IAU Colloq. 31. Reference should also be made to important review papers by Simonenko and Levin (11.105.141) on meteorite orbits and by Wetherill (11.105.105) on the source of meteorites and large meteoroids.

One of the trends noticed during the last three years is the increased interest in the rapid recovery of meteorites. To aid in tracking fireballs multiple camera networks have been set up in Canada, England, Germany and Czechoslovakia. Details of these programs are listed elsewhere in this report. An important by-product of the photographic programs is the determina- 
tion of precise orbits of bright meteors. The location of meteorite impact points by seismic methods has recently been proposed (12.105.036).

Visual recordings of meteors are reported at about the same level as in previous years. In the last few years there has, however, been an unfortunate decline in the number of radar and photographic programs. The Harvard Radio Meteor project and the Prairie Network photographic program have been terminated. At present few meteor radars are operating on a continuous basis. Attention is, however, called to the high level of activity in meteor radar studies maintained by Soviet investigators. Important papers on radar and photographic studies of meteors appear regularly in Vestn. Kiev. Univ. Ser. Astron., Bull. Inst. Astrophys. Akad. Nauk Tadzhik S.S.R., Bjull. Kom. Komet. Meteor. Akad. Nauk Tadzhik S.S.R. and other U.S.S.R. journals.

The existing multi-station camera networks have limited time coverage and can only record very bright meteors. Visual recording of meteors by experienced amateur groups is therefore of great importance - in particular for the detection of new meteor showers. Visual programs are particularly important at low latitudes and in the southern hemisphere, where our knowledge of the normal meteor background is very incomplete.

At the other end of the mass spectrum the in-situ measurements from rockets, satellites and space probes have produced valuable data on the interplanetary dust component. Statistics of lunar microcraters have produced information on the flux of microparticles over very long time periods. The direct space probe measurements have given information on the dust environment at large distances from the Earth $(12.106 .072,13.106 .037,12.106 .029,13.106 .026)$. The satellite and space probe measurements have resulted in a great increase in the number of publications relevant to our commission. Unfortunately a number of these reports are to be found in the non-astronomical literature and/or in technical reports of limited circulation.

There is an increasing amount of observational evidence to indicate that the near-Earth microparticle flux may differ significantly from that observed in interplanetary space $(12.105 .052,12.104 .062,13.104 .032)$. Renewed interest in this problem has been stimulated by measurements from the Heos and Prospero satellites (Bedford et al., 1975). Although theoretical models for an orbiting dust cloud are still being studied $(10.106 .016$, Coll. 13) most authorities agree that the circumterrestrial orbiting dust cloud model is untenable. This question has been reexamined by Anan'eva and Kostylev (09.104.022) and Lebedinets et al. (10.106.015). The authors conclude that an orbiting dust cloud can only be formed under very special conditions. One must therefore look for other explanations of the reported near-Earth flux enhancement. Amongst various mechanisms proposed are: breakup of large particles by an interaction with the Earth's atmosphere and ionosphere (Fechtig and Hemenway, Coll. 31); a flux contribution from ablation products of large fireballs (Ceplecha, Coll. 31). A difficulty in the atmospheric-interaction models is that the near-Earth flux enhancement appears to be observable at some distance from the Earth. This problem deserves further detailed study.

\section{REFERENCE}

Bedford, D. K., Adams, N. G., and Smith, D.: 1975, Planet. Space Sci. 23, 1451.

\section{PHOTOGRAPHIC METEORS}

\section{(Z. Ceplecha)}

\section{Fireball Networks}

Precise data on bright fireballs obtained from multiple station photographic programs have made possible significant progress in understanding the structure and composition of meteoroids. The most important of these programs is the Prairie Network (PN) operated by the Smithson. Astrophys. Obs. (Center for Astrophysics, Cambridge, U.S.A.) under the direction of McCrosky. The operation of the PN started in 1964 and was terminated on 1975, June 1. 
McCrosky, Posen and Shao are preparing a compilation of PN fireball trajectory data for publication in the Smithson. Astrophys. Obs. Spec. Rep.

A new fireball network is in continuous operation since 1971 under the direction of Halliday (Coll. 13, 12.105.019). The Meteorite Observation and Recovery Project (MORP) operates 12 stations routinely in western Canada. Since January 1973 it has recorded 450 bright meteors of which 125 were photographed at two or more stations. One event appears to be a possibility for a small meteorite fall. A prediscovery observation from one of the MORP stations of Nova Cyg 1975 on Aug 29 at $V=4.4$ on the ascending branch shows that fireball networks are capable of providing important observations for other objects than meteors.

The European fireball network (EN), consisting of 46 separate stations equipped with one all-sky camera each, has been in continuous operation: the Czech part was directed by Ceplecha (Ondřejov Observatory of ČSAV), the Slovak part by Porubčan (Astronomical Institute of SAV, Bratislava), and the German part by Kirsten (Max-Planck-Institut für Kernphysik, Heidelberg). A second independent part of the European Network started operations in England in 1973 under the direction of Hindley (Meteor Section of the BAA). In 1975 this system consisted of 21 separate stations with prospects for more than 30 in the near future. Each station is equipped with a $35 \mathrm{~mm}$ film camera with an auxiliary $f / 8$ fish-eye lens.

A new type of all-sky camera has been tested at Ondrejov Observatory. These cameras are gradually replacing the less precise old ones. A fish-eye objective Opton-Distagon 1:3.5, $f=30 \mathrm{~mm}$, field of view $180^{\circ}$, is used. The diameter of the image of the entire sky is $80 \mathrm{~mm}$ and the positional precision is one minute of arc. The camera records all meteors down to zero magnitude over the entire sky, and it is therefore capable of combining the photographic recording of fireballs and small-camera meteors into a joint program.

\section{Fireball Data Analysis}

Ceplecha and McCrosky (in prep., J. Geophys. Res., 1976) studied the structure and composition of 232 fireball bodies photographed by the PN. Several criteria (based mainly on the fireball end-height) were established to distinguish among different structural strength of meteoroids. The results were calibrated by using the Lost City fireball data. Three groups of fireballs were found: group I corresponding to ordinary chondritic stones, group II corresponding to weak carbonaceous chondrites and group III corresponding to still more fragile cometary material. Each of these groups contains approximately the same number of fireballs. Group III is further subdivided into group IIIa of cometary material of ordinary type and IIIb of Draconid shower type. Observational errors and natural dispersion in meteoroid structure produce severe smoothing effects. The end-height diagnostic is, in itself, not entirely convincing (especially the separation of I from II). However, one can discern differences in orbits and beginning-heights among the three end-height groups. Also Pribram fireball data are in agreement with these results. The above fireball groups were related to the beginning-height classes previously found by Ceplecha for faint meteors. The group II corresponds to the class $A$, the group IIIa to the class $\mathrm{C} 1$ (the class $\mathrm{C} 2$ of high velocity bodies is much less significant for fireballs than for fainter meteors), the group IIIb to the class 'greater than C1' (a notation used for faint meteors of Draconid shower type; I would recommend to use a symbol D in future papers). The photographic results do not exclude the possibility that group I corresponds to the densest bodies of class $A(I+I I \leftrightarrow A)$, but it is more probable that group $I$ corresponds to the very few so called 'asteroidal' meteors discussed by some authors. Thus the equivalent of the fireball group I seems to be almost absent for fainter meteors.

Information about the brightest fireballs photographed by the EN were published in the Event Notification Reports of the Center for Short-Lived Phenomena (Smithson. Inst.) and in Bull. Astron. Inst. Czech. (09.104.003, 13.104.003). One bright fireball observed on 1974, Aug 30 ('Leutkirch Fireball') was of the type $I$ and looked very promising for a meteorite search $\left(\max\right.$. abs. magn $=-10$, initial velocity $=12.6 \mathrm{~km} \mathrm{~s}^{-1}$, terminal height $=25.9 \mathrm{~km}$, terminal velocity $=4.2 \mathrm{~km} \mathrm{~s}^{-1}$, terminal mass $=14 \mathrm{~kg}$ ). However, no meteorite was found. Another fireball of 1974, Dec 4 ('Sumava Fireball') is the brightest fireball ever recorded by the EN (max. abs. magn. - 22). It is of type IIIb (terminal height $55 \mathrm{~km}$ with terminal mass virtually zero). Several spectra of this fireball were obtained at Ondrejov Observatory, the 
greatest dispersion achieved being $5 \AA \mathrm{mm}^{-1}$. The emission line spectrum does not differ from that known for fainter fireballs. A preliminary identification shows lines of Fe I, Ca I, Ca II, $\mathrm{Mg}$ I, Na I., Cr I, Mn I, Al I.

Rawcliffe et al. (11.104.009) and Jacchia (11.104.065) published data on a daylight fireball, which entered the atmosphere on 1972, Aug 10, at such small angle with the Earth's surface that it left the atmosphere to continue its travel around the Sun. Reference should also be made to two papers by Öpik (13.104.030) and Caulk (1975). The fireball traversed $1500 \mathrm{~km}$ of its luminous trajectory above the U.S.A. and Canada (Utah to Alberta) in more than $100 \mathrm{~s}$. The closest distance from the surface was $58 \mathrm{~km}$. The visible trajectory was photographed by many occasional photographers because of its long duration. Colour motion pictures of the fireball were taken. Satellite-borne infrared observations were available. (Most of the fireball photographs were gathered by Simmons, the regional coordinator of the American Meteor Society). Sonic booms were heard near the mid-trajectory. A typical short-period loweccentricity orbit was computed by McCrosky.

ReVelle $(1973,13.104 .004)$ has studied theoretically the sound phenomena associated with the penetration of large meteoroids deep into the atmosphere. Donn and Balachandran (12.104.007) believe they have detected acoustic sounds from meteors and estimated the trajectory (on the basis of an assumption of reasonable elevation). But there is a lack of independent evidence that their sources were in fact meteors (ReVelle, 1975). McIntosh et al. (1976) have recorded infrasound from a large meteor (believed to be a Geminid) detected by meteor radars. Meteor acoustic observations are as yet very scarce but McIntosh et al. have initiated a project of systematic infrasound observations in conjunction with the Canadian prairie fireball network.

\section{Other Programs}

Classical meteor photography with small cameras was performed in the U.S.A., in Canada, in Czechoslovakia and in the U.S.S.R.

Harvey (Langley Research Center, U.S.A.) has established a double-station direct-camera meteor patrol in New Mexico. It is operated by Cuffey from the New Mexico State University. This program yields geometrical and dynamical data for the meteor spectra photographed at the same location. Electrically operated K24 cameras, two at Tortugas mountain and two at the Corralitos site, require attention only once every ten days. The patrol cameras have operated for two years, giving 6000 one-hour exposures, with 260 meteor photographs. Orbits of 34 meteors have been obtained. The patrol is now yielding about 10 double-station meteors per month. Trajectory and orbit reductions of 20 double-station meteors have been completed by Tedesco and Harvey.

Ceplecha continued his systematic double-station meteor program using 30 small cameras with rotating shutters, placed at two locations $40 \mathrm{~km}$ apart. This program has been operated without interruption for $25 \mathrm{yrs}$. In about $8000 \mathrm{~h}$ of exposure time (equivalent to $240000 \mathrm{~h}$ exposure of one camera), 50000 photographic plates $(9 \times 12 \mathrm{~cm})$ have been exposed. About 1200 double-station meteors of good quality have been recorded. Most meteors are sporadic; the Príbram fireball is one of these. Trajectory and orbit reductions from 1951 to 1962 were completed and partially published. The 30 camera double-station program will be terminated in 1976. Instead a joint multi-station program for fireballs and meteors using Opton-Distagon fish-eye objectives will be put into operation. It is expected to yield good records of meteors down to zero magnitude. Supporting geometrical and dynamical information relating to the meteor spectra photographed at Ondreejov Observatory will thus be available with sufficient precision also after the termination of the classical multi-camera double-station program. Data reduction is continuing and the entire material on trajectories and orbits for all $25 \mathrm{yr}$ will hopefully be ready for publication at the end of this decade.

A Super-Schmidt meteor camera has been installed at Springhill Meteor Observatory near Ottawa, Canada. It is used to provide support for programs of meteor spectroscopy employing electronic image intensification.

Babadžanov and Khaimov (11.104.031) studied meteor flares observed by the double-station photographic program at Dushanbe, U.S.S.R. They found typical dimensions of flares to be 1 
to $2 \mathrm{~km}$, with a typical duration of 0.02 to $0.06 \mathrm{~s}$. The heights of flares increase and the duration of flares decrease with increasing velocity. The integrated brightness of the flare is proportional to the integrated light curve of the meteor.

Benyukh (09.104.019) studied bulk densities of meteoroids derived from photographic beginning heights. Shestaka (09.104.023) studied the dependence of meteor duration and position of maximum light on various parameters of the trajectory of photographic meteors. Simonenko (10.104.054) presented a generalized theory of meteor fragmentation and used it to explain inconsistencies in bulk densities for photographic meteors.

Using photographic data, Cook (1973) classified meteor showers into class A, B, C1 and C2 (classification system of Ceplecha). He associates class $C$ meteors with the residue of the iceimpregnated surface of a cometary nucleus after sublimation of ices, and class A meteors with the core of a cometary nucleus. Class B meteoroids are associated with less dense cores of smaller cometary nuclei (no meteor stream classified as B has a known comet). The density of class A meteoroids $\left(1.2 \mathrm{~g} \mathrm{~cm}^{-3}\right)$ is so close to that of type I carbonaceous chondrites as to suggest that the latter come from old cores of very large cometary nuclei. Cook et al. (1973) made some revisions of and additions to the newly found photographic meteor streams published by Lindblad (06.104.076-.077).

Kresáková (12.104.001) investigated errors in reciprocal semimajor axis, $1 / a$, for meteor orbits. She estimated an error of $\pm 0.01 \mathrm{AU}^{-1}$ for precise reduction of the best measurable photographic trails and $\pm 0.09 \mathrm{AU}^{-1}$ for graphical reduction of shorter trails. Kresák and Kresáková (12.104.047) showed that it is possible to obtain an upper limit of dispersion of $1 / a$ within one meteor stream, even if the dispersion due to observational errors considerably exceeds the real deviations from the parent comet's orbit.

A dependence of colour index of photographic meteors on velocity was found by Hajduková (12.104.050). Colour index increases with velocity by more than one magnitude over the observed velocity interval. This result contradicts previous results of other authors and points to the importance of proper division of data.

The problems of meteor luminous efficiency were studied in the laboratory by Becker and Slattery (10.104.059) and compared with results from photographic meteors. A generalized meteor luminosity equation was derived and compared with the classical equation by Ceplecha (10.104.006). The results were applied to 4 bright photographic meteors with good spectral records.

\section{Television Techniques}

Faint meteors were observed by means of television techniques. Clifton (10.104.031) from NASA Marshall Space Flight Center used the secondary electron conduction vidicon and was able to record meteors down to about +9 stellar magnitude for angular velocity of $10^{\circ}$ per second $\left(+11^{\mathrm{m}}\right.$ for stationary source). The dependence of the hourly rates on magnitudes, daily variations and other statistics are given. Jones and Hawkes $(11.104 .021,13.104 .005$, 13.104.010) observed faint meteors using an RCA 4589 vidicon, fibre-optically coupled to a 3-stage Varo 8585 image intensifier. This system gave $+8.5^{m}$ as a limit for a stationary source $\left(3^{m}\right.$ lower sensitivity than that of the Clifton system). Applying meteor theory and statistical methods to their observations, they found that the solid, compact meteoroid model must be abandoned. It is very important to extend the single-station TV observations into doublestation TV observations in the future, and thus to avoid some of the pitfalls of simple meteorcounting statistics.

\section{REFERENCES}

Caulk, H. M.: 1975, Astrophys. Letters 16, 99.

Cook, A. F.: 1973, Smithson. Contr. Astrophys. No. 14.

Cook, A. F., Lindblad, B. A., Marsden, B. G., McCrosky, R. E., Posen, A.: 1973, Smithson. Contr. Astrophys. No. 15.

McIntosh, B. A., Watson, M. D. and ReVelle, D. O.: 1976, Can. J. Phys. (in press).

ReVelle, D.O.: 1973, Acoustics of Meteors, Technical Report 010816 (3 parts), Univ. of Mich.

ReVelle, D. O.: 1975, Science 189, 394. 


\section{RADAR METEORS}

(R. B. Southworth)

As in the previous report, this section is primarily concerned with physical processes in the ionized column left by the moving meteoroid. Reliable interpretation of radar observations depends on adequate understanding of these processes, but the literature does not yet show any general agreement about them. It will be profitable to reanalyze a great many existing observations when such an agreement is reached. Even now, an internationally-agreed 'temporary standard physical model' for radar meteors would be valuable for intercomparison of results if investigators did not find too much labor in analyzing twice, once using the standard model and once using their own preferred models.

For conciseness, this section does not report meteor observations made to determine winds or atmospheric temperature or pressure; nor does it discuss equipment, or correlations of meteors with sporadic $E$. Orbits and streams of radar meteors are reviewed elsewhere. Nonetheless, the equatorial observations of Babadzhanov et al. (12.104.004, 12.104.012) are noted here because they circumvent some of the difficulties in interpretation of radar meteor data.

Kashcheev and Novoselova (1975) observed irregular diffraction patterns interpretable as fragmentation in at least 36 of 61 streams and associations; this confirms prior observations of fragmentation by others. Their earlier observation (10.104.037) of decelerations larger than expected for single-body meteoroids also tends to confirm large decelerations previously observed by others, even though they did not find the excess statistically significant (11.104.004). Further proof of fragmentation is given by recent television observations (13.104.010) of shorter light curves than are consistent with single-body meteors, again confirming earlier television and radar measures of trail length. Fragmented meteoroids will almost always give a reduced radar signal compared with a single meteoroid of equal mass, often severely reduced. There is an evident danger of selection effects that cannot be evaluated until more is known about the distribution of fragment masses and relative fragment velocities.

Studies of the early development of the ionized column include discussions of the intitial electron energies (6.104.024) and atom and ion diffusion cross-sections (11.104.016), as well as ambipolar diffusion of mixed species (6.104.014). The radial distribution of electrons was again observed (12.104.038) to fall off somewhat faster than in a Gaussian distribution. The effect of electron collisions on echo signal strength has been discussed (12.104.054). (The abstract does not do justice to the paper.) In a re-examination of radio echo theory, a new (quadrapole) plasma resonance has been discovered (12.104.002).

Two studies of the complex chemical processes in (primarily overdense) meteor trains have been published by Baggaley and Cummack (12.104.027) and Poole and Nicholson (1975, 12.104.031). These agree in concluding that dissociative recombination is much more important than attachment in removing electrons from the train, and in explaining differences between day and night train durations by changes in atmospheric constituents. A large number of prior studies of 'attachment' must probably be reconsidered. Regrettably, it seems likely that a full understanding must depend more on other studies than on radar meteor observations. New data on the change in character of head echoes over the day $(7.104 .004)$ is not yet explained.

Elements of enduring meteor trains that have spread away from the trajectory along the geomagnetic field have been observed (6.104.051) and explained (10.104.014). Alignment of the trajectory along the field did not cause any conspicuous change in the character of the radar echo.

The parameter $s$ of the mass distribution is, of course, important for comparison of rates observed using radars of different sensitivity, and even for rates for different velocities using the same radar, but it is not reliably known. Observations of diurnal $(10.104 .026,12.104 .058)$ and annual $(10.104 .025)$ variations in $s$ confirm that it is not a universal constant among radar meteors.

Lindblad (1976) has presented a very suggestive relationship between solar activity (probably X-rays) and variation in meteor rates, although Kennewell and Ellyett (12.104.026) 
argue that a superimposed effect of volcanic dust is necessary to explain the peak rates of 1963 . In either case, a decrease in atmospheric scale height, corresponding to a drop in temperature at meteor heights, would shorten meteor trails and therefore increase their brightness and linear electron density. No one has published a quantitative treatment; the decrease in the spread of meteoroid fragments consequent upon a shorter scale height may considerably magnify the direct effects of the scale height.

\section{REFERENCES}

Kashcheev, B. L. and Novoselova, N. V.: 1975, Astron. Vestnik 9, 3.

Lindblad, B. A.: 1976, Nature 259, 99.

Poole, L. M. G. and Nicholson, T. F.: 1975, Planetary Space Sci. 23, 1261.

\section{METEOR SPECTROSCOPY}

(P. M. Millman)

The quantities of observational data available in the field of meteor spectroscopy have changed dramatically in recent years. While only some two dozen records existed in the early thirties, and just a few hundred in the fifties, we can now list thousands of meteor spectra. For example, during the peak of the Geminid shower in December, 1974, well over one thousand records were secured by Clifton on four nights with two spectrographs operated by the NASA Marshall Space Flight Center in Arizona, USA. This remarkable advance in data acquisition has been a result of the systematic use of newly-designed spectrographic instruments capable of detecting much fainter meteors than had been possible in the past.

Observational programs involving the use of electronic image intensification in closed-circuit television-type systems have been continued in both the United States and Canada through cooperation among the Dudley Observatory, Albany, N.Y.; the Smithsonian Astrophysical Observatory, Cambridge, Mass.; the Marshall Space Flight Center, Huntsville, Ala.; and the National Research Council of Canada, Ottawa, Ont. (Millman and Clifton, 1975). At two stations near Ottawa some two dozen meteor spectrographs, utilizing conventional photography, are added to the television recording. Harvey at NASA Langley Research Center, Hampton, Va., is operating a battery of Maksutov-type meteor spectrographs in cooperation with the New Mexico State University. These observations are supplemented by a two-station direct-camera meteor patrol. An airborne expedition in October, 1972, resulted in two faint Giacobinid spectra, secured by the Langley Research Center team (10.104.028). Russell has continued his program of photographic observations of the spectra of the Perseid meteors, and has suggested correlations of spectral features with various physical parameters of the meteoroids or the atmosphere $(10.104 .029$, Coll. 31). Batteries of meteor spectrographs are currently used on active programs of photography at the Ondrejov Observatory. At the Ashkhabad Astrophysical Laboratory Begkhanov, Goulmedov and Moukhamednazarov (1975) have obtained grating spectra of faint meteors using orthicon and television equipment.

Detailed analysis of meteor spectra has been carried out at a number of research institutions. At Dushanbe in the U.S.S.R. Saidov and Zolova have published photometric analyses of 7 bright meteor spectra photographed in the period 1960-1964 (11.104.040, 11.104.041). Harvey has continued his studies of the large collection of spectra secured on the Langley systematic meteor patrol (11.104.048), and has noted the existence of a small fraction (about 1\%) of essentially iron-deficient or sodium-deficient meteoroids which may favor retrograde orbits (11.104.022). He has also carried out a photometric study of meteor spectra with strong features arising from $\mathrm{N}, \mathrm{O}$, and $\mathrm{N}_{2}$ (J. Geophys. Res., in press). Smirnov (11.104.002) and Saidov and Zolova (11.104.045) have estimated the color indices of meteors through photometry of their spectra. A more detailed study of the relations among size, brightness, velocity and spectral reddening of meteors has been made by Rajchl at Ondrejov (11.104.013). 
The interpretation of meteor spectra has been considered by several investigators. Ceplecha has proposed a model of the spectral radiation for typical bright fireballs $(10.104 .006)$, and Rajchl has discussed the possible origin of a puzzling long-duration feature in the violet region of wavelength (08.104.040). Kalenichenko at Kiev University has proposed that an excitation mechanism involving transfer from metastable states of $O$ I and $N$ I may strongly enhance certain lines in the violet and near-ultraviolet $(08.104 .029,08.104 .030)$.

The problem of the determination of meteoroid composition from the photometry of the meteor spectrum is still a difficult one, owing to the lack of laboratory studies of collision cross-sections and to gaps in our knowledge of the details in the physical theory of light radiation from meteors. Saidov has found an abundance by weight for $\mathrm{Na}$ of $6 \%$ in three meteor spectra (11.104.042). Yavnel has discussed the relation between the composition of chondrites and relative abundances of certain elements as estimated from meteor spectra (12.104.022). Some of the difficulties inherent in finding quantitatively the chemical composition of the meteoroid from a study of the corresponding meteor spectrum have been reviewed by Harvey (10.104.008), and by Millman (Coll.31).

In spite of the large quantity of observational data now available, as noted above, many important problems in this field remain to be solved. We may have some of the answers when more of the recently recorded spectra have been analysed in detail. As has often been said before, there is a crying need for further laboratory studies of the physical parameters of atoms and molecules under conditions that approximate those in the upper atmosphere.

\section{REFERENCES}

Begkhanov, M., Goulmedov, K. and Moukhamednazarov, S.: 1975, Izv. Akad. Nauk Turkm. S.S.R. Nos. 1 and 3 .

Millman, P. M. and Clifton, K. S.: 1975, Can. J. Phys. 53, 1939.

\section{PHYSICAL THEORY OF METEORS}

\section{(A. F. Cook)}

There are three current approaches to the physical theory of meteors. The first of these starts from the classical theory and seeks to evaluate the constants of that theory together with the luminous efficiency as a function of velocity. Laboratory experiments for the determination of luminous efficiencies of various elements have been made by Savage and Boitnott (Coll. 13) and by Becker and Slattery (10.104.059). Laboratory experiments for the determination of drag and heat transfer coefficients and luminous efficiencies have been reported by Friichtenicht and Becker (Coll. 13). A new method which uses two well-determined parameters involving the overall trajectory and end points of fireballs has been introduced by Ceplecha (1975) and Ceplecha and McCrosky (unpublished). Correction of an important error in the old determination of the dependence of colour index on velocity has been made by Hajduková (12.104.050).

The second approach introduces plausible complications to the classical theory in an effort to fit most observations of meteors. Discussions of the dustball model of a meteor have been published by Simonenko (12.104.006), Hawkes and Jones $(12.104 .015,1975)$ and Hughes (11.104.064). The small solid meteoroid with heat sink and cooling by surface radiation has been examined by Lebedinets (Coll. 13).

The third approach attempts a detailed explanation of artificial meteors or of a natural object of plainly similar character. This problem has been neglected too long. The paper by Griffin (1975) reports on the iron meteor of 1956 November 1 observed from Meanook and Newbrook and includes references to the literature on artificial meteors as well. This important paper should provide a challenge and an inspiration to those who would construct models of the meteoric process.

For very large meteors (fireballs, bolides, meteorite falls) the reader is directed to the comprehensive review by Bronstehn (12.105.040). 


\section{REFERENCES}

Ceplecha, Z.: 1975, Bull. Astron. Inst. Czech. 26, 242.

Griffin, A. A.: 1975, J. Roy. Astron. Soc. Canada 69, 126.

Hawkes, R. L. and Jones, J.: 1975, Monthly Notices Roy. Astron. Soc. 173, 339.

\section{METEOR ORBITS}

\section{(L. Kresák)}

The past triennium was relatively meagre on publication of new data on individual meteor orbits. After the Harvard Radio Meteor Program, also the operation of the Prairie Network for determining photographic orbits of bright fireballs was discontinued. Lost City has remained the only recovered meteorite recorded during the ten years of operation of the network, but a very extensive material on fireball orbits has been collected which awaits further analysis.

Statistical evaluation of orbital data on meteors obtained by different techniques was mainly concerned with the discrimination of minor and diffuse meteor streams. The earlier ad hoc identifications, brought to a more objective basis by the introduction of Southworth's D-criterion, have evolved into computerized searches of non-random clusterings in a phase-space of orbital elements. Such an approach makes it possible to allow for the inference of the sporadic background, with its complex variable features. Sekanina $(09.104 .004,1975)$ has used a combination of three vectorial elements with a subsequent application of the D-criterion to identify meteor streams in a sample of over 19000 radio orbits. Sosnova and Lebedinets (09.104.021) have developed a searching scheme using four basic elements: $1 / a, e, i$, and $\widetilde{\omega}$. A search by Lebedinets et al. (09.104.018) among nearly 17000 double-station radio orbits allowed to discriminate 715 meteor groupings about one half of which seems to refer to real streams and associations. These were found to contribute $30 \%$ to $40 \%$ of all meteors of magnitude 6.0 to 7.5 , as compared with a minimum estimate of $7.5 \%$ by Sekanina. A detailed stream search amongst 1667 Southern Hemisphere radio meteor orbits has been published by Gartrell and Elford (1975). Cook et al. (1973) have extended the search in the largest existing sample of 2400 photographic orbits by McCrosky and Posen. A case of chain associations among meteor showers and a comet, involving the Geminid stream, was investigated by Kresáková (11.104.012).

Based on radio observations of 37000 faint meteors, Kashcheev et al. (10.104.040) have determined the distribution in individual elements. Particularly noteworthy is their low value of the perihelion distance of maximum occurrence, about $q=0.3$. Fedynskij et al: $(11.104 .015)$ relate a model distribution of sporadic orbits to the observed diurnal variation of orbital elements. Radio data on velocities and radiants of 4500 meteors observed near the equator, at Mogadiscio, Somalia, were used by Babadzhanov et al. (12.014.004, 12.104.013) to establish the differences between the distribution of meteor orbits in the northern and southern hemisphere. The increasing prevalence of direct motions with decreasing particle size was confirmed by Nazarenko (12.104.057).

The accuracy of meteor orbits from different photographic surveys was examined by Kresáková (12.104.001). Within the range of observational uncertainty there is no difference in mean semimajor axis between shower meteors of different brightness, or between the meteors and their parent comets, which could be attributed to the Poynting-Robertson effect. A method of separating the spread due to measuring errors from the virtual dispersion of meteor orbits was developed by Kresák and Kresáková (12.104.047). An application to 300 photographic Perseid orbits showed that for a majority of orbits the actual range of the revolution periods, $100<P<170 \mathrm{yr}$ is much narrower than indicated by the observations which include as much as 30\% of spurious hyperbolic orbits. Hajduk et al. (12.104.014) and McIntosh (12.104.049) presented another method of determining the revolution periods of meteor streams, based on the yearly variation of meteor rates. For radar Geminids, McIntosh obtained $1.50<P<1.65 \mathrm{yr}$, in good agreement with the photographic measurements. The reliability of 
the orbits of meteorites determined from visual observations was critically examined by Simonenko and Levin (11.105.141).

While the attainable accuracy of individual meteor orbits does not admit any reasonable computation of the effects of planetary perturbations, meteor streams provide a variety of possibilities for modelling the dispersive effects. The ejection velocities from the parent comets were estimated by Katasev and Kulikova (11.104.059) on the basis of the orbital data. They find less than $2.5 \mathrm{~m} \mathrm{~s}^{-1}$ for the Leonids, $50 \mathrm{~m} \mathrm{~s}^{-1}$ for the Draconids, $100 \mathrm{~m} \mathrm{~s}^{-1}$ for the Perseids, and $250 \mathrm{~m} \mathrm{~s}^{-1}$ for the Taurids - but one can question whether the higher values are not overestimated, due to a high age of the streams. Kulikova (11.104.051) even finds $350 \mathrm{~m} \mathrm{~s}^{-1}$ for the Taurids. The rapid dispersion following a close approach to Jupiter was investigated by Sherbaum $(10.104 .044,12.104 .052)$; the wavy disturbances emanating from the original orbit were shown to dissolve completely within a few revolutions if the miss distance is of the order of $0.1 \mathrm{AU}$. Detailed computations of perturbations of the $\alpha$-Virginid stream for a time period of $200 \mathrm{yr}$ by Kazimirchak-Polonskaya and Terent'eva (Coll. 22, 09.104.026) illustrate the evolution of low inclination meteor orbits meeting Jupiter near aphelion. The results indicate a rapid rotation of the nodal line, oscillations in eccentricity and perihelion distance, and essential stability of the other elements. The impact of the orbital evolution on the observed characteristics of meteor showers (visibility, duration, position, size and shape of radiant areas) is examined, and it is suggested that even the puzzling presence of northern and southern branches of some meteor streams can be explained by Jupiter's perturbations.

The possibility of capture of meteoroids into circumterrestrial orbits by deceleration at atmosphere-grazing passages was investigated by Anan'eva and Kostylev (09.104.022). The conclusion is that if the passage occurs at a low geocentric velocity at a specific height, the transition into a circumterrestrial orbit is possible, and that this orbit may remain stable over as much as $100 \mathrm{yr}$.

Incidentally, the first large fireball detected by a satellite-borne infrared radiometer (11.104.009) and widely photographed from the west of U.S.A. during its day-time appearance on 1972, August 10, was travelling in a grazing trajectory with a minimum height of about $50 \mathrm{~km}$. The orbital elements computed by McCrosky (11.104.065) do not essentially differ from the majority of the Prairie Network data: the perihelion was near the Earth's orbit and the aphelion in the inner part of the asteroid belt. Another exceptional object, possibly the brightest fireball ever photographed, was recorded by the European All-Sky Network on 1974, December 4. The orbital computation by Ceplecha (Coll. 31) gave an aphelion distance of 3.5 AU. This is far inside the orbit of Jupiter, in spite of the unusual Draconid type of the meteoroid which is tentatively interpreted as indicating fresh cometary material.

A different kind of atmospheric interaction was considered by Mitra (11.082.009) who suggested that the superrotation of the Earth's atmosphere above $150 \mathrm{~km}$ could be due to the global deposition of meteoric matter. However, strong arguments against this explanation were put forward by Hawkes (13.082.013), who showed that the process of momentum transfer would take place much lower. McIntosh (13.082.062) showed that observational evidence on the distribution of meteor orbits does not support Mitra's suggestion - it even tends to point to an opposite effect to that required by Mitra.

Further evidence in favour of the asteroidal origin of meteorites has been put forward by Anders (13.105.080). He finds that the amount of trapped solar-wind gases in gas-rich meteorites is only compatible with parent bodies which remained long enough in the asteroid region to develop a substantial regolith. This argument also involves the carbonaceous chondrites which were believed to represent the most probable candidates for a cometary material recovered on the Earth. By the way, a carbonaceous-chondritic nature seems to be very common among the main belt asteroids (Chapman et al., 13.098.050).

An interesting possibility of injection of asteroidal fragments into Earth-crossing orbits was pointed out by Zimmerman and Wetherill $(10.105 .039,11.105 .085)$. They show that almost one half of the bodies ejected randomly from the $2: 1$ resonance gap of Jupiter, at velocities of 100 to $200 \mathrm{~m} \mathrm{~s}^{-1}$, would experience close approaches to the Earth on a time scale of $10^{4}$ to $10^{5} \mathrm{yr}$. The orbits of these hypothetical asteroidal fragments would match very well to the 
observed orbits of many meteors and fireballs. A comparable injection mechanism based on the presence of secular resonances was proposed by Williams (1973).

\section{REFERENCES}

Cook, A. F., Lindblad, B. A., Marsden, B. G., McCrosky, R.,E., and Posen, A.: 1973, Smithson. Contr. Astrophys. 15, 1.

Gartrell, G. and Elford, W. G.: 1975, Austral. J. Phys. 28, 591.

Sekanina, Z.: 1975, Icarus (in press)

Williams, J. G.: 1973, Eos Trans. Amer. Geophys. Union 54, 233.

\section{METEOR SHOWERS}

\section{(B. A. Lindblad)}

\section{A. Photographic, Radar, and Theoretical Studies}

In the period 1972-75, several investigations concerning the formation and evolution of meteor streams have been published. The concept of a 'jet stream' was introduced by Alfvén (03.107.001), and further studied by Baxter and Thompson (10.061.001). A recent summary of these problems has been given by Trulsen (Coll. 31). The relevance of the jet stream hypothesis to the comet-meteor stream complex has been considered by Mendis (1973) and Ip and Mendis (12.106.017).

The problem of the dissipation of meteor streams has claimed the attention of a number of workers. Astapovich (10.104.049) has computed the life times of meteor showers. Katasev and Kulikova (09.104.016) have investigated the evolution of a meteor stream under the influence of the Yarkovsky-Radzievsky effect. Times of fall into the Sun for various particle sizes and spin conditions are given. From a study of photographic meteor orbits Kramer and Musij (10.104.036) have compared the dispersion in inverse semi-major axis and in the Tisserand constant for a number of meteor streams. No large difference between the two dispersion measures was found, indicating that gravitational perturbations by Jupiter play only a minor role in the dispersion of stream orbits. In a series of papers Adamiak (08.104.049), Karmiński $(11.104 .062)$ and Gaska $(08.098 .043,1974)$ have examined the dispersion of the inclination of photographic and radio meteor orbits. The results are presented in $\sigma(i)-a$ diagrams. Their studies indicate a systematic variation in $\sigma(i)$ with semi-major axis of the orbit.

Collision- and solar system ejection probabilities at close encounters of small bodies to planets have been investigated by Weidenschilling (1975). Barracelli (10.098.008) has considered how close encounters with small bodies affect a planetary orbit and investigated under which circumstances this disturbance is comparable in magnitude to disturbances caused by secular perturbations. Sherbaum (12.104.052) has critically examined the disturbance on a meteor stream of a close approach to Jupiter.

The density of meteoroids belonging to various streams has been examined in photographic meteor data by Benyukh $(09.104 .036,11.104 .052)$. A strong dependence of meteoroid density on the type of the orbit is evident. From a review of Harvard photographic meteor data Lindblad (13.104.017, Coll. 31) concludes that meteoroid density for short period orbits increases with decrease in se mi-major axis. From radio echo data Verniani (10.104.058) derives a median density of $0.8 \mathrm{~g} \mathrm{~cm}^{-3}$. This suggests for the radio meteors a porous structure similar to that found in photographic meteor data. However, Bibarsov and Chebotarev (11.104.066, 1975) claim higher values of density for radio meteors and also find that density increases with decrease in mass.

\section{Comet-Meteor Stream Associations}

Kulikova (11.104.051) applies numerical models to investigate the association of the Taurid meteor stream with comet Encke. The best agreement between the present Taurid orbit and the 
model is obtained if it is assumed that the meteoric material was ejected at a point in the comet orbit where the true anomaly is about $80^{\circ}-90^{\circ}$, i.e. far from perihelion.

The orbit of comet 1862 III (Swift-Tuttle) has been redetermined by Marsden (10.103.107). A comparison of Marsden's orbit with the mean orbit of photographic Perseid meteors has been made by Kresák and Kresáková (12.104.047). The theoretical radiant associated with comet Kohoutek (1973f) has been computed by Terenteva $(10.104 .039,11.104 .028)$. High-power radar observations of a meteor shower with the predicted radiant coordinates have been reported by Kashchejev et al. (12.104.033).

The first comprehensive computer search for meteor streams and comet-meteor stream associations in the Southern Hemisphere using radio meteor data has been made by Gartrell and Elford (1975). Predictions for a southern hemisphere meteor shower associated with comet Grigg-Skjellerup were presented by Ridley (07.104.021). An increase in meteor radar rates, which was attributed to comet Grigg-Skjellerup, was reported by Baggaley (09.104.007).

\section{B. Visual and Telescope Observations}

\section{Visual}

Active programs of visual meteor observations were continued at a number of locations in the U.S.A., Canada, Great Britain, the Netherlands, Sweden, Czechoslovakia, U.S.S.R., India and Japan. Visual observations were collected by the American Meteor Society (AMS) as in previous years (Olivier et al., 1971-73). A third catalogue of Northern Hemisphere daily hourly rates covering the period 1964-72 has been compiled. A new information bulletin 'Meteor News' is published by an amateur group in Jacksonville, Florida. Several amateur groups report on new meteor stream radiants $(11.104 .019,11.104 .070)$. Visual meteor observations have been made by groups in Japan (08.104.048, 08.104.054). Rao et al. $(11.104 .071,12.104 .029,1974)$ have presented results of an extensive study of minor meteor showers observed during the winter months 1961-72 at Waltair, India (lat. 17 .5N). Identified shower radiants are found to lie close to the ecliptic plane.

The Meteor Section of the British Astronomical Association (BAA) has organized a meteoric recovery network under the direction of Hindley (1975). More than 200 fireballs were reported to the meteor section in one year. Visual sitings of fireballs have been reported in BAA Circulars (13.104.038) and in JBAA (07.104.022-023).

A critical analysis of visual meteor data was made by Hughes (11.104.007) who discussed the incident flux of visual meteors brighter than $0 m$. In analysing meteor rates, from which we compute the meteoroid flux, a basic problem is to determine the collection area of a visual observer. It is noted that this area may vary by a factor of ten depending on the type of program which is being carried out.

Štohl and Millman (10.104.034) published a detailed analysis of the errors in visual magnitude estimates. A systematic difference is found between the magnitude scales of the Ottawa and Skalnate Pleso teams - the Ottawa estimates are systematically brighter by $0.5-1.0$ mag. The discrepancy may be partly caused by the better air-transparency at the Skalnate Pleso mountain site. The Springhill mean magnitude $(\bar{m}=2.00)$, however, appears to be some what low compared with the mean magnitude of other visual programs.

\section{Telescopic}

Extensive programs of observing telescopic meteors have been conducted by the Meteor Section of the Czechoslovak Astron. Soc. (Kučera et al., 1975). The program emphasizes the flux determination of telescopic meteors, the structure of telescopic meteors, the structure of telescopic meteor radiants, and the slope of the luminosity curve. Porubčan (09.104.001) has examined the telescopic radiant areas of the Perseids and Orionids. Telescopic observations of Perseid meteors have been reported by Vladmirov and Nicolov (11.104.030). Statistics of brightness and trail length are presented.

\section{Other Programs}

The program of simultaneous visual, telescopic and radar observations of meteors was 
continued at Ondrejov, Czechoslovakia. Radar, photographic and visual meteor programs at the Springhill Meteor Observatory are concentrated to periods of shower activity. The observational program now includes image orthicon meteor spectroscopy (13.104.035). Combined radar and visual recordings of meteors have been continued by Lindblad (Coll. 31). The AugustSeptember radar data now cover a period of more than 20 yr. Previously reported long-term variations in radar rates of a solar cycle origin are confirmed in the recent data.

Large fluctuations in the impact counts of dust detectors onboard a lunar orbiter have been observed by Alexander et al. (12.106.043). Over a time period of four years count rate increases appear to correlate with activity of major meteor showers. The increases in rates are ascribed to lunar ejecta from stream meteoroids which impact on the lunar surface.

\section{Individual Showers}

\section{Quadrantids}

Radio observations of the Quandrantid shower are described by Isamutidinov (07.104.020), Hughes $(07.104 .001, .036)$ and Poole et al. (07.104.003). A study of the Quadrantid incident flux based on radar observations in 1966-74 is presented by Belkovich and Tokhtasev $(11.104 .027,12.104 .051)$. The nodal regression of the stream has been investigated by Hughes (07.104.019).

\section{Eta Aquarids}

Observations of this shower have been reported by Thackeray (10.104.015) and Clark (10.104.060). Hajduk $(09.104 .002)$ has studied the variation in meteor rates at successive shower returns. The study supports the concept of a common origin of the Eta Aquarid and Orionid streams associated with comet Halley (13.104.019).

\section{Perseids}

The structure of the Perseid radiant is investigated by Batylova et al. (08.104.015), and Martynenko et al. $(09.104 .020, .025,10.104 .013)$. Visual observations by amateur groups in Holland and Belgium have been reported (09.104.006, 09.104.008).

\section{Draconids}

As noted in the previous report the major Draconid display predicted for 1972, October 8 was not observed. Meteor observations during this period have been reported by Millman (09.104.009), Fast and Litvinova (09.104.032), Bogdanov and Mikhajlov (09.104.035), Hughes and Thompson (10.104.002), Harvey (10.104.028), Khotinok (11.104.006), Simek (11.104.014), Terent'eva and Tryashin (11.104.020), and Saito and Tomita (11.104.063). The dust content of the upper atmosphere at the expected time of maximum of the Draconids has been studied by the twilight technique by Fehrenbach et al. (10.104.011).

\section{Taurids}

Fragmentation effects in Taurid meteors have been studied by Babadzhanov and Getman (13.104.037).

\section{Orionids}

Radar echo data obtained by the Ondrejov radar in 1961-69 at the time period of the Orionid shower are reported by Hajduk (13.104.031). Statistics of meteor head echoes observed during the Orionids are presented (07.104.004).

\section{Leonids}

The general conditions of an encounter with Leonid meteoroids has been discussed by Astapovich and Terent'eva (08.104.055). The structure of the Leonid return of 1969 has been investigated by Porubčan (12.104.048) using the radio observations at the Springhill Meteor Observatory. The flux of meteors in the range from $-1^{m}$ to $5^{m}$ during the 1966 return was 
studied by Chigorin (10.104.012). Hajduk (1973) has presented statistics of head echoes observed during the 1965 return.

VIII. Geminids

The meteoroid mass distribution in the Geminid stream has been described by Simek (10.104.004, 11.104.047, 13.104.001) and McIntosh and Šimek (11.104.049). A detailed comparison of the Ottowa and Ondrejov radar rates during the Geminid period has been made by Hadjuk et al. (12.104.014).

\section{Cyclids}

The Cyclid meteor stream, detected by Southworth and Hawkins (1963), has an orbit similar to that of the Earth. Possible members of this stream complex have been detected in radio searches by Sekanina (1975). Terent'eva $(10.104 .050)$ lists ephemerids of possible geocentric radiants. If the Cyclid stream should be considered as a physical entity or only represents unrelated orbits with a very high probability of detection is still an open question.

\section{REFERENCES}

Bibarsov, R. and Chebotarev, R: 1975, Astron. Vestnik, 9, No. 2.

Gartrell, G. and Elford, W.G.: 1975, Austral. J. Phys. 28, 591.

Gaska, S.: 1974, Bull. Astron. Obs. Toruń No. 54.

Hajduk, A.: 1973, Bull. Astron. Inst. Czech. 24, 330.

Hindley, K.B.: 1975, J.Br.Astron. Assoc. 85, 150.

Kučera, J., et al.: 1975, Contr. Obs. Planetarium Brno No. 19.

Mendis, D. A.: 1973, Astrophys. Space Sci. 20, 165.

Olivier, C.P. et al.: 1971-73, Amer. Meteor Soc. Reports Nos. 193-5.

Rao, M.S.: 1974, Current Sci. (India) 43, 647.

Sekanina, Z.: 1975, Center for Astrophys. Preprint No. 313.

South worth, R., Hawkins, G.: 1963, Smithson. Contr. Astrophys. 7, 261.

Weidenschilling, S.J.: 1975, Astron. J. 80, 145.

\section{METEORITE CRATERS}

\section{(I. Halliday)}

The study of terrestrial craters associated with meteorite impact continues to be an active field of research in various parts of the world. New features are being added each year to the list of generally accepted hypervelocity impact structures as field expeditions followed by laboratory studies reveal the various types of deformation produced in rocks by shock waves from high-velocity meteorite impacts. Intercomparison of this shock metamorphism among craters of different sizes and craters formed in different target rocks is leading to an understanding of the detailed mechanics of crater formation by the impact process. The dating of large craters permits estimates to be made of the cratering rate during different geological eras and the values obtained for the past billion years can be reconciled with the results of studies of young lunar craters. Impact craters sometimes provide a means of reconstructing ancient geological history and can be used to derive erosional rates on old continental land masses.

Although many technical papers on impact structures are to be found in the geological rather than astronomical literature, frequent references to work in this field may be found in Meteoritika (U.S.S.R. Academy of Sciences, in Russian) and in Meteoritics (The Meteoritical Society, published by Arizona State University). Masaitis (11.105.122) has summarized the studies of several craters in the U.S.S.R. and describes the nature of shock deformations found at the Kara, Boltysh, Puchezh-Katun, Janisjarvi, Kaluga, Mishina Gora and Ilinet craters. Masaitis et al. $(09.105 .158,13.105 .133,1975)$ have published a thorough study of the huge Popigay crater in eastern Siberia. Papunen (1973) has described the shock effects found at the Sääksjärvi crater in Finland while French et al. (1974) described similar effects from two 
structures in southeastern Libya. Robertson and Grieve (13.105.096) described definite impact evidence for 9 Canadian craters, bringing the total in Canada to 21. Two members of their list of probable craters, Slate Islands and Ile Rouleau (in Lake Mistassini) have since been confirmed, raising the total to 23. The large Araguainha Dome structure in Brazil, described by Dietz and French (10.105.020) contains evidence of shock metamorphism while drilling at Lonar Lake in India has revealed an abundance of similar effects $(09.105 .077,09.105 .141)$.

Dence (09.105.159) has published a significant review of terrestrial impact structures, stressing the relationships among the various morphological forms of fresh and eroded craters. Valuable data has been obtained from recent core drilling projects at the Barringer (Roddy, 1975), Ries (Schmidt-Kaler, 1974) Boltysh and Lonar Lake craters. Petroleum exploration drilling has also revealed evidence of buried impact structures in the western plains of North America (Sawatzky, 1975).

Smaller impact pits and craters produced by relatively low-velocity meteorite impacts continue to receive detailed attention. Krinov $(12.105 .039)$ has analyzed the complex pattern of Sikhote-Alin craters caused by successive fragmentation events during the atmospheric passage of the meteorite. Surveying and excavation of the Campo del Cielo field has been described by Romaña and Cassidy (11.105.069) while Hodge and Wright (11.105.017) have studied the distribution of meteoritic material around the Boxhole crater. Vasilyev et al. $(12.105 .047,13.105 .104)$ have collected and analyzed particles associated with the Tunguska event.

\section{REFERENCES}

Dence, M.R.: 1972, Proc. 24th. Int. Geol. Congress, Montreal, Sect. 15, 77.

French, B.M., Underwood, J.R., and Fisk, E.P.: 1974, Bull. Geol. Soc. Amer. 85, 1425.

Masaitis, V.L., Mikhailov, M.V., and Selivanovskaya, T.V.: 1975, Popigay Meteorite Crater, All-Union Scienti-

fic Research Geological Institute, Moscow.

Papunen, H.: 1973, Bull. Geol. Soc. Finland 45, 29.

Roddy, D.J.: 1975, Proc. VI Lunar Sci. Conf. (in press).

Schmidt-Kaler, H.: 1974, (ed.), "Die Forschungsbohrung Nördlingen" 1973, Geol. Bavarica 72, 5.

\section{METEORS AND THE EARTH'S ATMOSPHERE}

\section{(B.A. Lindblad)}

A number of investigators have studied the interrelation between meteors and various phenomena in the Earth's atmosphere. Optical manifestations of aerosols of assumed meteoric origin have been the subject of numerous investigations. Groundbased or balloon-borne measurements of the twilight sky have been reported by Fehrenbach and Link (12.082.088), Link (13.1 04.033), Divari et al. (10.082.085, Coll. 13), and others. Increases in the scattering coefficient is found in the upper atmosphere during the activity of meteor showers. Measurements of the brightness of the twilight sky may thus offer a possibility of monitoring the dust content of the upper at mosphere.

Meteor recordings may also provide important information about the aeronomy of the upper atmosphere. Hughes and Baggaley (07.104.036) studied the effect of sunrise on the meteor region. The vertical propagation of atmospheric tides was studied at Garchy (France) by Follous et al. (11.082.022) using meteor trails. Hughes $(1974,1975)$ and Lindblad (Coll. 31) have discussed the influence of solar activity on meteor rates and heights. These studies may provide information on the time variation of the density scale height at the meteor ablation level.

A new meteor radar system for the study of upper atmosphere temperature and density at Budrio (Italy) has been implemented by Verniani et al. (1974). Barnes (1973) has published a status report on radar meteor wind and density measurements. Both reports include extensive references. An automatic wind radar station is described by Kashev and Nechitajlenko $(13.033 .001, .002)$. At the Charkov Institute of Radioelectronics Kashev and coworkers have 
put into operation a new automatic radar equipment for determination of velocities, radiants and orbital elements of 12 th magnitude meteors.

Sen and Saha $(10.104 .030,13.104 .026)$ have investigated the effect of meteor showers on the propagation of radio signals. Correlations between ionospheric sporadic- $E$ phenomena and meteors are studied by Donij et al. (10.104.046), Latipov (11.104.032, 11.104.043, 12.104.059) and Tř́sková (1974). D-region absorption effects on meteor forward-scatter signals have been observed by Jones and Collins (10.104.032).

Poultney (12.104.061) has computed the arrival times and locations of cometary micrometeoroid influxes into the Earth's atmosphere. The possibility of observing this dust in the upper atmosphere is considered. A possible realtionship between cometary dust and rainfall singularities has been suggested by Kvíz (private comm.).

\section{REFERENCES}

Barnes, A.: 1973, Bull. Amer. Meteor. Soc. 54, 900.

Hughes, D.W.: 1974, Nature 252, 191.

Hughes, D.W.: 1975, COSPAR meeting, Space Res. (in press).

Trísková, L.: 1974, J. Atmospheric Terrest. Phys. 36, 861.

Verniani, F. et al. : 1974, Bull. Ass. Geofisica Italiana 23, 243.

\section{INTERPLANETARY DUST}

\section{(R.K. Soberman)}

It has become traditional to have a technical meeting in which micrometeoroids are a major subject approximately every two or three years. The latest of these was IAU Colloquium No. 31, which was co-sponsored by Working Group 3C (Cosmic Dust) of COSPAR. This Colloquium, "Interplanetary Dust and Zodiacal Light", was held June 10-13, 1975, in Heidelberg. The interrelationship between the photometric studies of the zodiacal light and the direct measurements of the interplanetary dust responsible for the phenomenon was highlighted by the meeting. The proceedings will be published in 1976.

During the COSPAR meeting in Constance, Germany, on 24 and 25 May, 1973, a symposium was held on "Noctilucent Clouds and Interplanetary Dust". The proceedings were published in Space Res. 14. There were two other regular meetings of Working Group 3C during the COSPAR sessions is São Paulo, Brazil in 1974, and in Varna, Bulgaria in 1975. The proceedings of those meetings will be published in Space Res. 15 and 16. For further information regarding the material presented in this summary, the reader is recommended to the Space Research series and to the proceedings of IAU Colloquium No. 31.

Alvarez (Coll. 31) described the results obtained from the penetration detectors on the Pioneer- 10 and -11 spacecraft. These results showed a near constant spatial concentration of 10 and $20-\mu$ particles from $1 \mathrm{AU}$ to beyond Jupiter. An anomalous gap in the dust concentration beginning at $1.16 \mathrm{AU}$ and extending to $2.3 \mathrm{AU}$ for the larger particles was noted in these measurements. The explanation of these gaps in the penetration data remains uncertain. A significantly increased concentration of particles was found in the vicinity of Jupiter. $(11.104 .010,12.104 .021,13.106 .026)$. While the analysis of the data is proceeding, the Langley researchers and Singer believe that the increased concentration can be accounted for gravitationally without invoking trapped orbits about the planet. Results obtained from the Meteoroid Technology Satellite in near-Earth orbit were presented by Alvarez (Coll. 31). These were highlighted by measurable fluxes of particles down to $10^{-17} \mathrm{~g}$. The mass distribution curve now appears to flatten out for submicron particles, but the existence of significant numbers of particles below the radiation pressure cut-off limit in the solar system has been established.

Berg (12.106.044, Coll. 31) summarized nearly 10 years of data obtained from the Pioneer-8 and -9 interplanetary spacecraft. He has measured fluxes of the order of $2-4 \times 10^{-4}$ particles per square meter per second in hyperbolic orbits coming from the solar direction. Zook (1975) has 
applied the term "beta meteoroids" to these particles signifying that the ratio of solar light pressure to gravitational attraction is greater than one. The prevailing view for the origin of these beta meteoroids is that they result from collision and/or evaporation of interplanetary dust which has come into the near-Sun vicinity due to the Poynting-Robertson effect. Whipple (Coll. 31) supports a collisional origin but at distances greater than $0,5 \mathrm{AU}$ from the Sun. Lamy (11.106.037, 12.106.023, Coll. 31) had devoted considerable theoretical effort to showing that sublimating particles can orbit in close proximity to the Sun, thus justifying a dusty component of the corona to $2 R_{\odot}$. The hypothesis of Hemenway $(08.082 .001$, Coll. 31) that particles of refractory materials can originate in the vicinity of sunspots is not generally accepted. Most investigators feel that this hypothesized source contributes few if any particles to the interplanetary complex.

The highly eccentric Earth-orbiting Heos A2 satellite also showed significant numbers of submicron particles. As reported by the Heidelberg group, they measured a general enrichment of a factor of 5 in the interplanetary dust within $10 R_{\Theta}$. They also measured an anisotropy of approximately one order of magnitude between the direction of the apex of the Earth's motion and the general background (13.106.007). There was a reported clustering of impact events. They termed one portion "groups", which they believe to be the result of secondary material ejected from the Moon. The other classification, "swarms", they believed to be the result of larger particles broken up in the vicinity of the Earth. Lastly, they reported (Coll. 31) that seven particles measured between 1974, May 16 and June 23 were believed to be ejected from comet Kohoutek (1973f).

The clustering of micrometeoroid events and the near-Earth enhancement was invoked by Bedford et al. $(12.104 .036,13.105 .146,1975)$ to explain the high fluxes measured by the instrument flown on the Prospero satellite. Kresák (Coll. 31) has shown that streams of small particles cannot persist in heliocentric orbits, but he does not rule out atmospheric fragmentation or lunar ejecta streams. Models of the dust component in near-Earth and interplanetary space have been worked out by Lebedinets (13.106.002) and Divari and Krilova (1973).

In contradiction to the Mie theory, the zodiacal light is now attributed to sunlight reflected from dust particles larger than $50 \mu$. This was concluded from the shape of the micrometeoroid mass distribution curve. Matsumoto $(10.106 .062$, Coll. 31) has shown that some of the polarization properties can be explained by Fresnel reflection, but further theoretical work is required on the optical properties of irregular dust particles. The photometric data obtained from Pioneer 10 and from the Helios space vehicles indicate that the cross-sectional area of the zodiacal light particles must decrease as the inverse first power of heliocentric distance. No such constant decrease has been noted in the spatial concentration of particles smaller than $20 \mu$. Evidence for a changing size distribution which could account for the decrease in total crosssectional area with heliocentric distance was presented by Soberman (12.106.029. Coll. 31) from his Pioneer-10 discrete-particle optical detector. These optical results also indicate that the particles are rotating rapidly and throwing off "glints" of light. Paddack (12.104.009, Coll. 31) has hypothesized that interplanetary dust particles spin up due to the action of solar radiation to the point where they self-destruct in a time short compared to their Poynting-Robertson lifetime in the solar system. That the particles do spin is generally conceded, but whether the particles can achieve an angular velocity sufficient for self-destruction remains in question. Also, on the subject of light-scatter from particles, Levasseur and Blamont (10.106.033, Coll. 31) have reported that short-period increases in the zodiacal light associated with meteor streams were measured from the D2-A satellite.

The dust experiments flown on the Pioneer- 10 and -11 spacecraft have established that there is no large increase of small particles in the asteroid belt. The enhancement reported was one that increased with increasing particle size as compared with the $1 \mathrm{AU}$ model. The origin of the inner solar system dust complex remains clouded. While $10-\mu$ particles persist beyond Jupiter, those responsible for the zodiacal light become negligible outside the asteroid belt. Delsemme has reported that comets could maintain the required 10 tons per second only if most of the mass were released in particles larger than $100 \mu$.

Programs for the collection of micrometeoroids have remained active. Recently, high altitude balloons which can circumnavigate the Earth have been used for such collections. The Dudley 
Observatory Program continues to collect particles from balloons and has also obtained collections from the Skylab manned space station (13.106.045). Some of these particles have low electron transparency, which they have interpreted as refractory materials coming from the Sun. Brownlee et al. (12.106.054, Coll. 31) have obtained particles from high altitude aircraft and balloon collections in what is believed to be pristine extra-terrestrial micrometeoroid condition. Credence is added to this belief by elemental composition studies which indicate normal cosmic abundances such as those found in carbonaceous chondrites. Many of these collected particles exhibit a surface fine structure of the order of $1000 \AA$.

Analysis of the lunar microcrater results have continued to engender controversy. Investigators disagree on whether there has been a measureable change in the micrometeoroid flux over the recent history of the Moon. Hartung and Storzer (12.094.820), Hartung (Coll. 31) and Ashworth and McDonnell (12.105.076) believe that the flux has been increasing over the past $10^{5}$ yr. Anders and Morrison (Coll. 31) disagree. There is agreement that there is no evidence for a radiation pressure cut-off at micron size, and several investigators are finding agreement between the lunar crater and satellite-determined fluxes. The craters are similar to those produced in the laboratory and give evidence of having been produced by particles with a composition similar to that of carbonaceous chondrites. A cumulative mass flux distribution has been derived from the Apollo window results and from the Surveyor 3 TV shroud data (12.094.234).

Some old controversies in the interplanetary dust field continue to arise. Roach (13.106.004, Coll. 31) has presented data from the OSO-6 zodiacal light experiment which provides evidence for the backscatter of light from the Earth-Moon libration regions. His data indicate movement of the bright patch, which he feels is the reason it may have escaped the notice of other investigators. Rosinski (Coll. 31), Rosinski et al. (09.105.040, 1975) have presented new evidence for a connection between rainfall peaks and magnetic spheroids of extraterrestrial origin.

Early results have been reported for two very recent experiments. Berg et al. (12.106.060, Coll. 31) report results from the detector which was left on the surface of the Moon by Apollo 16. Data from this instrument (which is similar to the one flown on Pioneers 8 and 9) indicate anomalous events at lunar sunrise and sunset. Rhee (Coll. 31) believes this is the result of migration of charged lunar dust at the terminator. The other experiment of interest is the micrometeoroid impact mass spectrometer which is on the Helios Sun probe. Data had been obtained from this instrument by the Heidelberg group but at the time of this writing no results are available.

\section{REFERENCES}

Bedford, D., Adams, N., and Smith, D: 1975, Planetary Space Sci. 23, 1451.

Divari, N.B. and Krilova, S.N.: 1973, Astron, Circ. No. 778.

Rosinski, J., Nagamoto, C., and Bayard, M.: 1975, J. Atmospheric Terrest. Phys. 37, 1231.

Zook, H.A.: 1975, Planet. Space Sci. 23, 1391.

Space Res. 14, Rycroft, M.J. and Reasenberg, R.D. (eds.) 1974.

Space Res. 15, Rycroft, M.J. (ed.) 1975.

Space Res. 16, Rycroft, M.J. (ed.) 1976.

B.A. LINDBLAD

President of the Commission 